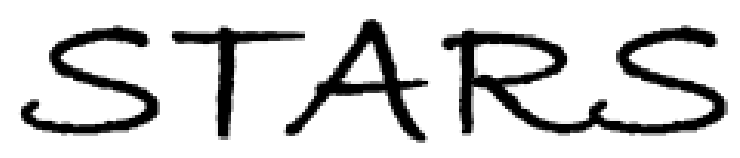

University of Central Florida

STARS

Faculty Bibliography 2010s

Faculty Bibliography

$1-1-2011$

\title{
Polymer translocation induced by a bad solvent
}

Christopher Lörscher

University of Central Florida

Tapio Ala-Nissila

Aniket Bhattacharya

Find similar works at: https://stars.library.ucf.edu/facultybib2010

University of Central Florida Libraries http://library.ucf.edu

This Article is brought to you for free and open access by the Faculty Bibliography at STARS. It has been accepted for inclusion in Faculty Bibliography 2010 s by an authorized administrator of STARS. For more information, please contactSTARS@ucf.edu.

\section{Recommended Citation}

Lörscher, Christopher; Ala-Nissila, Tapio; and Bhattacharya, Aniket, "Polymer translocation induced by a bad solvent" (2011). Faculty Bibliography 2010s. 1586.

https://stars.library.ucf.edu/facultybib2010/1586

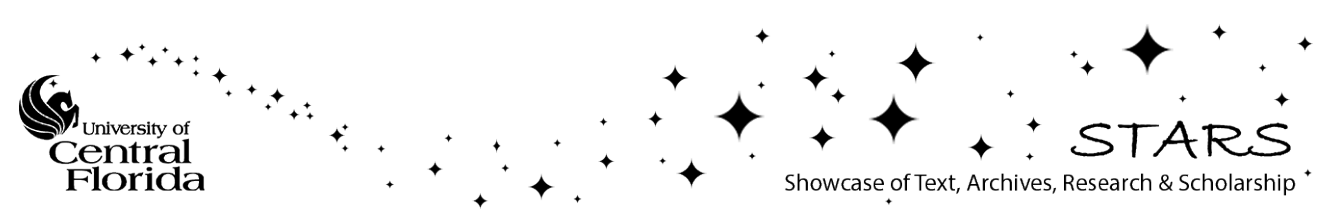




\title{
Polymer translocation induced by a bad solvent
}

\author{
Christopher Lörscher, ${ }^{1}$ Tapio Ala-Nissila, ${ }^{2,3}$ and Aniket Bhattacharya ${ }^{1, *}$ \\ ${ }^{1}$ Department of Physics, University of Central Florida, Orlando, Florida 32816-2385, USA \\ ${ }^{2}$ Department of Applied Physics, Aalto University School of Science, P.O. Box 11000, FI-00076 Aalto, Espoo, Finland \\ ${ }^{3}$ Department of Physics, Box 1843, Brown University, Providence, Rhode Island 02912-1843, USA \\ (Received 8 June 2010; revised manuscript received 12 November 2010; published 26 January 2011)
}

\begin{abstract}
We study polymer translocation through a nanopore subject to conformational differences created by putting two different solvents at the cis and trans compartments using Langevin dynamics in three dimensions (3D). Initially a fraction of the chain is placed in a good solvent at the cis side and the rest of the chain at the trans side is immersed in a bad solvent where it forms a globule. We study several aspects of the translocating chain as a function of the strength of the interaction $\varepsilon / k_{B} T$ for the bad solvent, where the temperature $T$ is kept below the $\Theta$ temperature for the specific bead-spring model that we have used to describe the chain. For $\varepsilon / k_{B} T \geqslant 1$ we find the mean first passage time $\langle\tau\rangle \sim\left(\frac{\varepsilon}{k_{B} T}\right)^{-1}$ and $\langle\tau\rangle \sim N^{1.1 \pm 0.05}$. In that regard, translocation under solvent asymmetry is similar to the case of driven translocation under a bias inside the pore. However, the globule formed at the trans in the immediate vicinity of the pore readily absorbs the incoming particles making the translocation process faster than the driven translocation. Our simulation results for long chains and $\varepsilon / k_{B} T \geqslant 1$ agree well with a theoretical prediction by Muthukumar [M. Muthukumar, J. Chem. Phys. 111, 10371 (1999).].
\end{abstract}

DOI: 10.1103/PhysRevE.83.011914

\section{INTRODUCTION}

Translocation of biomolecules through a small pore in a membrane is a common but important phenomenon in all living matters [1]. The entry and exit of DNA or RNA through pores in cell and nuclear membranes triggers other essential activities in these systems. About a decade ago in a series of experiments through a $\alpha$-hemolysin protein pore it was demonstrated that not only the mean first passage time (MFPT), but the shape of the histogram of the MFPT depends on the nature of the interaction of the polymer with the pore [2-5]. This important observation immediately generated other experimental studies to control the motion of the chain through the pore by using magnetic tweezers and other devices with an aim to develop fast and cheap DNA sequencing kits. Quite naturally, these experimental studies provided enough impetus for further theoretical [6-20] and simulation studies [21-37].

There have been quite a number of insightful theoretical studies pioneered by Sung and Park [6,7], Muthukumar [8,9], Chuang, Kantor, and Kardar [10], Kantor and Kardar [11] in terms of the reaction coordinate $s$ whose time evolution is governed by a Fokker-Planck equation containing free energy due to entropy of the chain at the cis and trans compartments. Dubbeldam and coworkers approached the problem using a fractional Fokker-Planck equation characterized by an exponent for anomalous diffusion $[12,13]$. These approaches are justified for slow translocation where the relaxation time is comparable to the translocation time. However, for biased translocation where the translocation time is typically much faster than the relaxation time, a number of simulation studies reported the out of equilibrium aspects of the translocating chain [35-37]. Under a bias the chain conformations not only show large variations, but the conformations at the trans side is very different from those at the cis side.

\footnotetext{
*Author to whom the correspondence should be addressed: aniket@physics.ucf.edu
}

PACS number(s): 87.15.A-, 87.15.H-, 36.20.-r

Driven translocation under a bias has been studied by a number of authors using a bead-spring model of homo and heteropolymer chains using Monte Carlo [13,23] and molecular dynamics methods [22,28,36,37]. Using Langevin dynamics simulation on a coarse-grained bead-spring model it has also been demonstrated as a proof of principle that the residence time of the translocating beads oscillates with a definite pattern that depends on the specific pore-monomer interaction from which the sequence of the chain can be predicted $[24,25]$. Langevin dynamics simulation has also been carried out to study translocation through a pore while one end of the chain is being pulled with a force [26]. One of the central questions that has been asked in the community is how the MFPT $\langle\tau\rangle$ depends on the chain length $N$ characterized by the translocation exponent $\alpha$ defined as $\langle\tau\rangle \sim N^{\alpha}$. It is now accepted that for driven translocation this exponent is not universal and for a narrow pore and a thin wall whose linear dimension and width are of the order of the size of the individual beads, respectively, the translocation exponent $\alpha$ lies within $2 v<\alpha<1+v[17,28,36]$. The upper limit $\langle\tau\rangle \sim N^{1+v}$ is the prediction of Kantor and Kardar [11] in the limit of small bias where one assumes the gyration radius $R_{g} \sim N^{v}$ and the velocity of the center of mass of the chain $v_{C M} \sim 1 / N$, so that $\langle\tau\rangle \sim R_{g} / v_{C M} \sim N^{1+\nu}$.

In the present work we study yet another interesting problem where the translocation is driven by differences in conformational statistics of the chain segments residing at the cis and the trans compartments arising out of the different solvents in each compartment. More specifically, we study the case here where the polymer at the cis side resides in a good solvent and is characterized by the Flory exponent $v=0.59$ in three dimensions (3D) for a self-avoiding random walk chain [38], while the polymer encounters a bad solvent at the trans compartment where the translocated segments form a globule. The polymer segments upon entering the tras side get immediately adsorbed and become part of the globule. The globule grows in size until the last bead translocates. The globule at the trans side acts as a ratchet which promotes 
directional translocation. Other types of ratchets, such as, Brownian ratchets, have also been discussed in the literature in the context of an efficient but generic method of translocation $[6,39,40]$.

While drawing an analogy with the case of voltage driven translocation we find interesting differences. In the case of voltage driven translocation where both the cis and trans side are in the good solvent the primary limiting factor for translocation is the formation of a high density region by the translocated beads in the immediate vicinity of the pore. In the case of the bad solvent, since the translocated segments are immediately adsorbed to become part of the existing globule, we find the translocation is faster in this case. The present simulation study is a special case of the theoretical study of translocation due to Muthukumar [8] where it was shown that any asymmetry in the average conformation of the polymer across the pore is sufficient to generate the driving force for the translocation. We compare our results with this theoretical prediction.

\section{MODEL}

We use the Langevin equation to study the Brownian motion of particles in a solution where the equation of motion for each monomer at position $\mathbf{r}_{i}$ is described by a stochastic differential equation

$$
m \ddot{\mathbf{r}_{i}}(t)=-\nabla U_{i}-\Gamma \dot{\mathbf{r}}_{i}(t)+\mathbf{W}_{i}(t),
$$

where the total interaction

$$
U_{i}=U_{\mathrm{FENE}}^{i}+\sum U_{L J}^{i j},
$$

is the sum of the finitely extensible nonlinear elastic (FENE) spring potential interaction [41] between two successive beads in the chain

$$
U_{\mathrm{FENE}}\left(r_{i j}\right)=-\frac{1}{2} k R_{0}^{2} \ln \left[1-\left(\frac{r_{i j}}{R_{0}}\right)^{2}\right],
$$

and the Lennard-Jones (LJ) interaction between any two neighboring monomers

$$
U_{\mathrm{LJ}}\left(r_{i j}\right)=4 \varepsilon_{i j}\left[\left(\frac{\sigma_{i j}}{r_{i j}}\right)^{12}-\left(\frac{\sigma_{i j}}{r_{i j}}\right)^{6}-\left(\frac{\sigma_{i j}}{r_{i j}^{c}}\right)^{12}+\left(\frac{\sigma_{i j}}{r_{i j}^{c}}\right)^{6}\right] .
$$

The term $\mathbf{W}(t)$ describes the influence of Markovian white noise due to the solvent, which is not taken into account explicitly here. It satisfies the fluctuation-dissipation relation

$$
\langle\mathbf{W}(t) \cdot \mathbf{W}(\tau)\rangle=6 k_{B} T \Gamma \delta_{i j} \delta(t-\tau),
$$

where $k_{B}$ is the Boltzmann constant and $T$ is the temperature of the bath. To model asymmetric solvent conditions at the cis and trans sides, first we label the particles with index 1, 2 , or 3 if they are located at the cis side, inside the pore, or at the trans side, respectively, and introduce a $3 \times 3$ cutoff matrix whose elements are either $2^{1 / 6} \sigma$ or $2.5 \sigma$ as follows. For all the monomers at the cis and trans sides $r_{c}^{11}=2^{1 / 6} \sigma$ and $r_{c}^{33}=2.5 \sigma$, respectively. We choose $r_{c}^{13}=2^{1 / 6} \sigma$, but due to the presence of the wall, this implies that the monomers at the cis and trans sides do not interact with each other. We index the monomers residing inside the pore according to their horizontal distance from the pore in increasing order. The rightmost monomer inside the pore sees the bad solvent so that $r_{c}^{23}=2.5 \sigma$. The rest of the monomers see the good solvent so that $r_{c}^{13}=r_{c}^{22}=2^{1 / 6} \sigma$. The cutoff matrix is symmetric. Typically for the parameters chosen we find there are no more than two monomers inside the pore. Likewise, the interactions among all the monomers are characterized by a $3 \times 3$ symmetric matrix $\epsilon_{i j}$ whose elements are set to unity for all the elements excepting $\epsilon_{33}=\varepsilon \epsilon_{11}$, where the parameter $\varepsilon$ is varied during the simulation.

The purely repulsive wall consists of one monolayer of immobile LJ particles of diameter $1.5 \sigma$ on a triangular lattice at the $x y$ plane at $z=0$. The pore is created by removing the particle at the center. The polymer beads interact with the wall particles with the same LJ potential with a cutoff distance $2^{1 / 6}\left(\frac{\sigma+1.5 \sigma}{2}\right)=1.25 \cdot 2^{1 / 6} \sigma$. The reduced units of length, time, and temperature are chosen to be $\sigma, \sigma \sqrt{\frac{m}{\epsilon}}$, and $\epsilon_{11} / k_{B}$, respectively. For the spring potential we have chosen $k=30$ and $R_{i j}=1.5 \sigma$, the friction coefficient. $\Gamma=1.0$, and the temperature is kept at $T=2.0 / k_{B}$ throughout the simulation. The $\Theta$ temperature for this model for this set of parameters $\Theta \simeq 4.0 / k_{B}$ [42]. Therefore, this ensures that the monomers at the trans compartment are immersed in a bad solvent. For a chosen fraction of the monomers at the cis/trans [Figs. 1(a)-1(c)] we equilibrate the chain for a time on the order of the Rouse relaxation time $\tau \sim N^{1+2 v}$, where the Flory exponent $v=0.588$ in $3 \mathrm{D}$ [38]. The chain is then
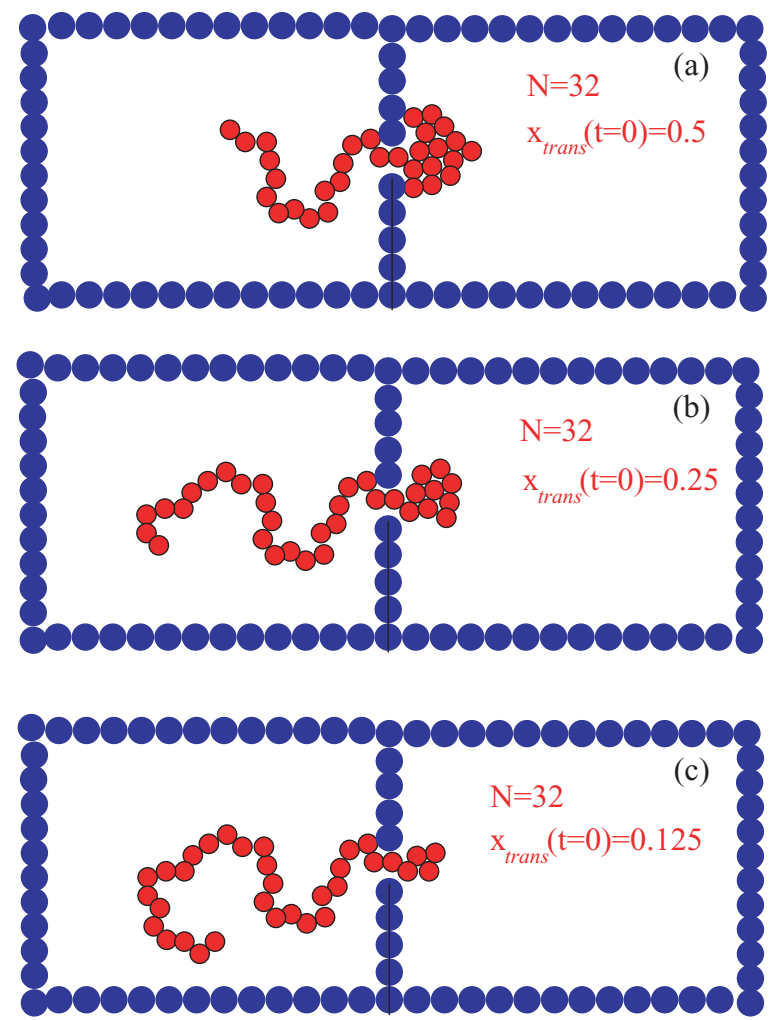

FIG. 1. (Color online) Depiction of the initial configuration of the chain of length $N=32$ to be translocated. At $t=0$ (a) $50 \%$ $\left[N_{\text {trans }}(t=0)=16\right]$, (b) $25 \%\left[N_{\text {trans }}(t=0)=8\right]$, and (c) $0.125 \%$ $\left[N_{\text {trans }}(t=0)=4\right]$ of the chain is on the trans side. These configurations are equilibrated up to Rouse relaxation time with the monomer inside the pore being pinned at the center of the pore before allowing the chain to translocate. 

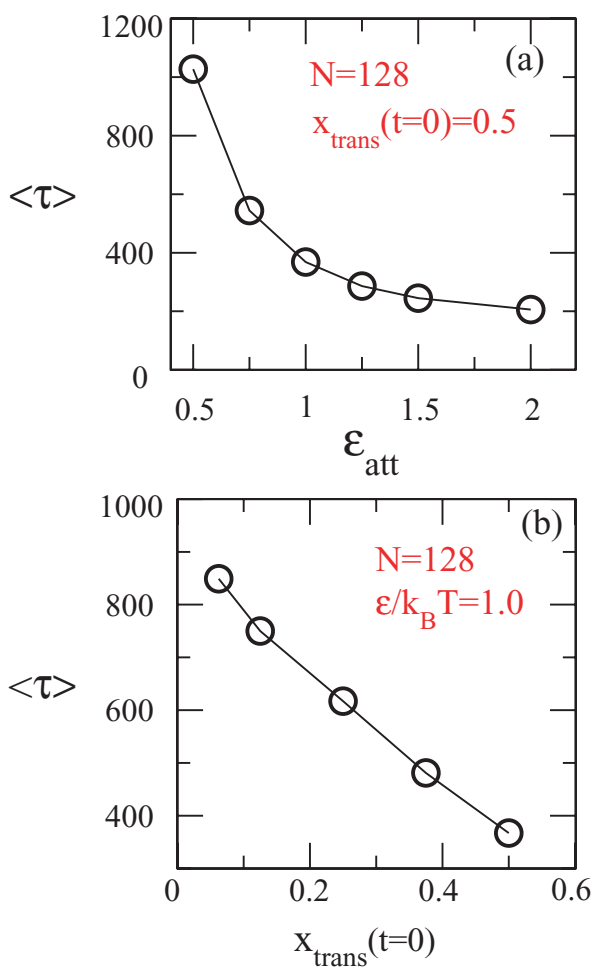

FIG. 2. (Color online) Mean translocation time $\langle\tau\rangle$ for a chain of length $N=128$ as a function of (a) the attractive strength $\varepsilon / k_{B} T$ for $x_{\text {trans }}(t=0)=0.5$ and (b) as a function of $x_{\text {trans }}(t=0)$ for $\varepsilon / k_{B} T=$ 1.0 to show how $\langle\tau\rangle$ increases as the interaction strength and $x_{\text {trans }}(t=0)$ are lowered.

allowed to translocate using a time step of $d t=0.005$. As the last bead exits the pore, a translocation event is completed and the process is repeated for 2000 times for averaging. The free energy landscape with these different cutoff values for the interactions favors translocation from the cis to the trans side. We study how this effective chemical potential difference introduced through the parameter $\varepsilon / k_{B} T$ drives the system toward a faster translocation.

\section{RESULTS AND THEIR INTERPRETATION}

\section{A. Initial configuration of the chain}

Evidently, the translocation time for a chain is a function of the number of monomers on the trans side at the beginning of the translocation process $N_{t r}(t=0)$. Ideally to calculate the first passage time and hence $\langle\tau\rangle$ one would like to place the first monomer of the chain at the pore and monitor the translocation time. But for $\varepsilon / k_{B} T \lesssim 1$, having only a few monomers at the trans side $\langle\tau\rangle$ becomes very large as shown in Figs. 2(a) and 2(b). Therefore, to overcome this problem we have studied translocation for three different values of $x_{\text {trans }}(t=0)=0.5$, 0.25 , and 0.125 and systematically extracted the results for large chain length in the limit $x_{\text {trans }}(t=0) \rightarrow 0$. While this strategy may not be universally valid, this has been possible for this specific case because of steady, single file translocation for the major fraction of the chain as will be discussed in the next sections. The initial conditions are depicted in Figs. 1(a) through (c).
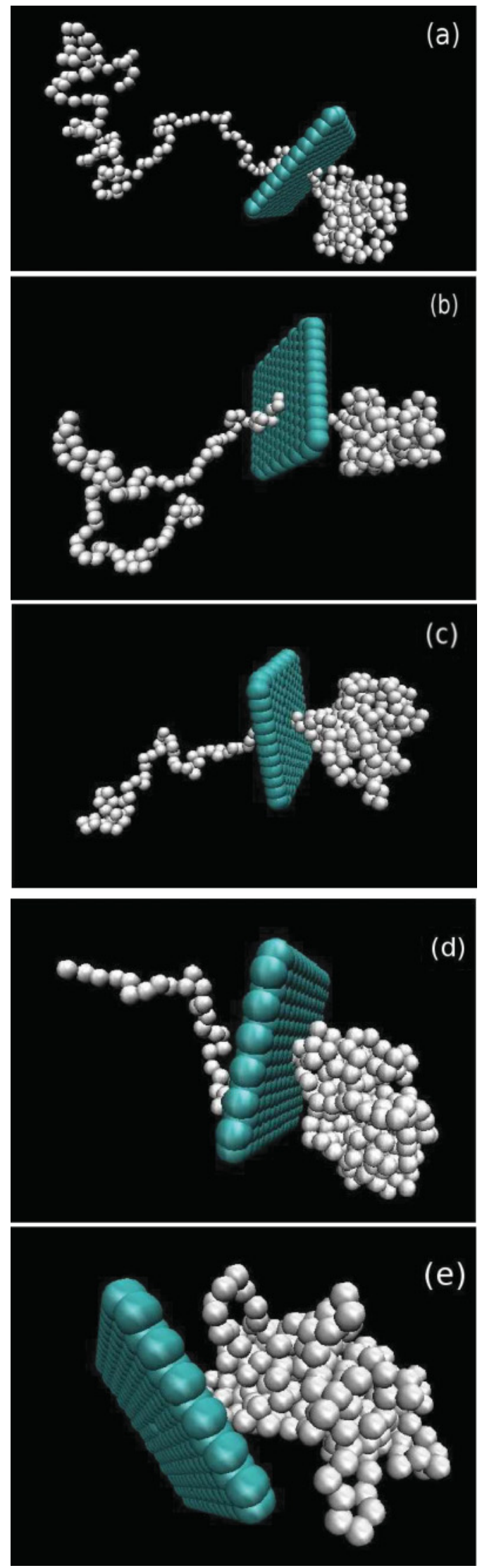

FIG. 3. (Color online) Snapshots of a translocating chain of length $N=256$ for $\epsilon / k_{B} T=0.5$ at times (a) $t=0$, (b) $0.25 \tau$, (c) $0.5 \tau$, (d) $0.75 \tau$, and (e) $1.0 \tau$, respectively. 

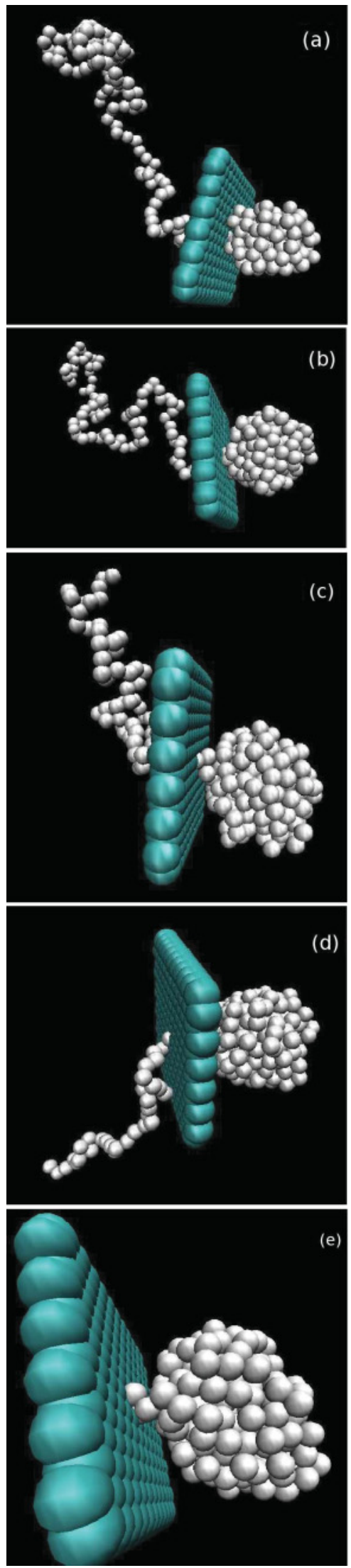

FIG. 4. (Color online) Snapshots of a translocating chain of length $N=256$ for $\epsilon / k_{B} T=1.5$ at times (a) $t=0$, (b) $0.25 \tau$, (c) $0.5 \tau$, (d) $0.75 \tau$, and (e) $1.0 \tau$, respectively.

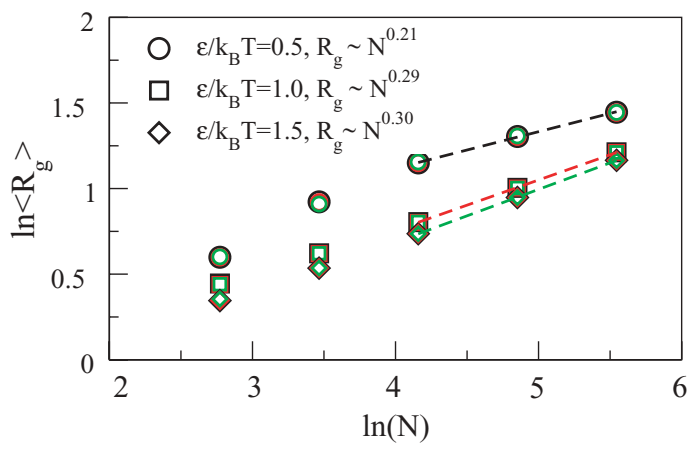

FIG. 5. (Color online) Variation of $\left\langle R_{g}\right\rangle$ as a function of chain length $N$ immediately after the completion of the translocation process for $\varepsilon / k_{B} T=0.5$ (circles), $\varepsilon / k_{B} T=1.0$ (squares), and $\varepsilon / k_{B} T=1.5$ (diamonds). In each case the different colors black, red, and green correspond to $x_{\text {trans }}=0.5,0.25$, and 0.125 , respectively, which are almost indistinguishable from each other.

\section{B. Translocation snapshots}

To get an idea of the translocation process, we show typical snapshots of a translocating chain in Figs. 3 and 4 at different stages of the translocation process. At $t=0$, the fraction of the chain that is located on the cis side is characterized by the equilibrium Flory exponent $v \simeq 0.588$ that corresponds to the good solvent condition. Since the trans part of the chain is in a poor solvent and the temperature is below the $\Theta$ temperature [42], it will form a globule which is expected to grow as a function of time. Comparing the snapshots for $\varepsilon / k_{B} T=0.5$ (Fig. 3) and $\varepsilon / k_{B} T=1.5$ (Fig. 4), we note that the globule formed by the translocated segments becomes more compact as the strength of the interaction increases. We have checked the $N$ dependence of the radius of gyration for chains immediately after the translocation process shown in Fig. 5. We find that for $\epsilon / k_{B} T=0.5, \epsilon / k_{B} T=1.0$, and $\epsilon / k_{B} T=1.5$ the gyration radius is $R_{g} \sim N^{0.21}, R_{g} \sim N^{0.29}$, and $R_{g} \sim N^{0.30}$, respectively. It is worth mentioning that if all the globules for different chain lengths were perfect spheres and fully relaxed then $R_{g} \sim N^{0.33}$. That the exponents are less than 0.33 indicates that the chains do not form a compact globule and/or the trans side of the chain does not have sufficient time to relax during the translocation process as discussed earlier in the literature [18,29,35,37].

\section{Characteristics of the monomers inside the pore}

One of the key questions that has been raised in polymer translocation is how the average translocation time $\langle\tau\rangle$ depends on the chain length $N\left(\langle\tau\rangle \sim N^{\alpha}\right)$. As indicated in the Introduction the exponent $\alpha$, even for a fully flexible chain, can depend on various factors. We know from previous studies that for diffusive translocation $\alpha=1+2 v$ while for forced translocation $\alpha \leqslant 1+v$. For the case studied here we find that for long chains and large interaction strengths $\alpha \simeq$ $1.10 \pm 0.05$. To gain a physical understanding in the following section we show results for the average monomer waiting time $W(m)$, the $z$ component of the monomer velocity $v_{z}(m)$, and the $z$ component of the LJ part of the force on the monomer $f_{z}^{\mathrm{LJ}}(m)$, respectively. We will see shortly that these quantities will reveal the translocation mechanism in operation. 


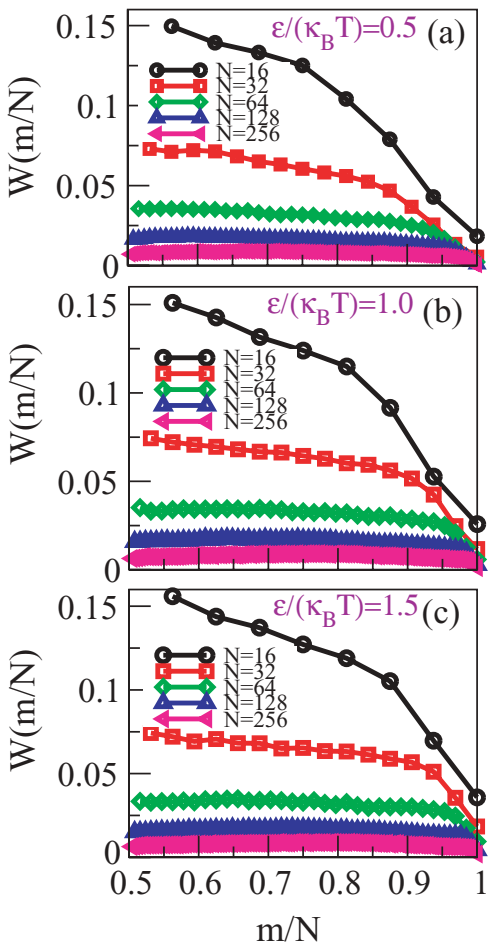

FIG. 6. (Color online) Average waiting time $W(m)$ as a function of monomer index $m$ for various chain lengths. The symbols circles (black), squares (red), diamonds (green), triangle up (blue), and triangle left (magenta) correspond to the chain lengths $N=16$, 32, 64, 128, and 256, respectively; (a) for $\varepsilon / k_{B} T=0.5$, (b) for $\varepsilon / k_{B} T=1.0$, and (c) for $\varepsilon / k_{B} T=1.5$, respectively.

\section{Residence time $W(m)$}

Figure 6 shows the average waiting time of those monomers which were at the cis compartment at $t=0$. To show the results for various chain lengths on the same scale, we have normalized the monomer index $m$ by the corresponding chain length $N$. The waiting time $W(m)$ is defined as the fraction of the total time a monomer having index $m$ spends inside the pore

$$
W(m)=\frac{\langle\tau(m)\rangle}{\langle\tau\rangle} .
$$

Therefore, by definition

$$
\sum_{m=1}^{N} W(m)=\sum_{m=1}^{N} \frac{\langle\tau(m)\rangle}{\langle\tau\rangle}=1 .
$$

The notation $\langle\cdot \cdot\rangle$ indicates ensemble average over 2000 iterations. We notice that there is a qualitative change for the waiting times for short and long chains. For very short chains (such as for $N=16$ and 32) $W(m)$ decreases monotonically. For longer chains $W(m)$ becomes relatively flat except for the last $1-2 \%$ of the beads for which the residence time quickly drops almost to zero. We observe that for a very long chain $W(m)$ becomes a constant except for the last few monomers at the end (Fig. 6). However, a more careful look reveals that for longer chains there is a slight increase in the residence time as a function of the bead index $m$, which then decreases beyond a certain value of $m_{\max }$ as shown in Fig. 7 for $x_{\text {trans }}(t=0)=0.5$ and for $\varepsilon / k_{B} T=0.5$. The dotted lines in Fig. 7 indicate the

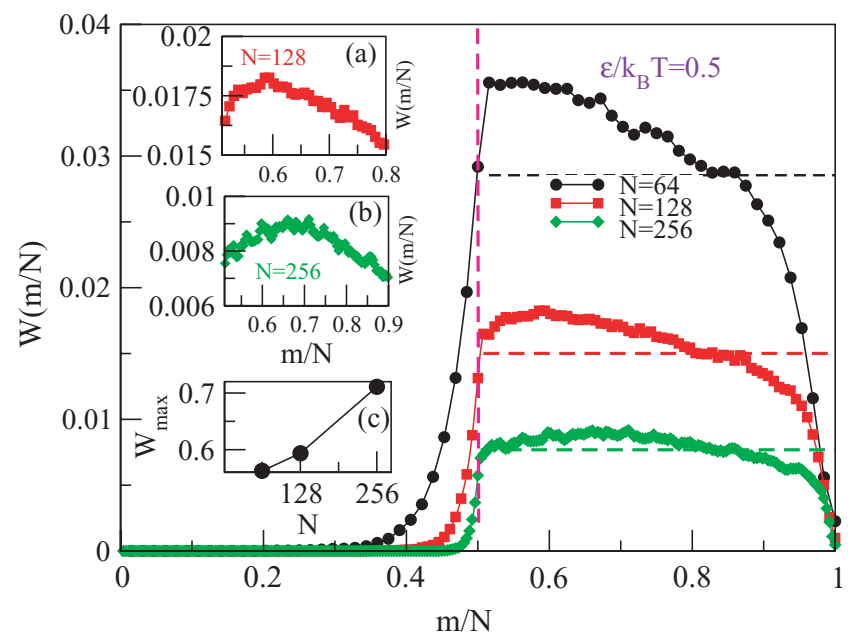

FIG. 7. (Color online) Average waiting time as a function of the monomer index for various chain lengths for $\varepsilon / k_{B} T=1.5$ and $N_{\text {trans }}(t=0) / N=0.5$ shown for the entire chain to demonstrate that $W(m)$ decreases rapidly for the monomers which are originally at the trans side at $t=0$. The nonzero values of $W(m)$ for $m<N_{\text {trans }}$ $(t=0)$ is attributed due to the back and forth motion of the beads during the translocation process. The insets (a) and (b) demonstrate that $W(m)$ has a maximum, and (c) shows that the positions of these maxima shift at higher values for longer chains.
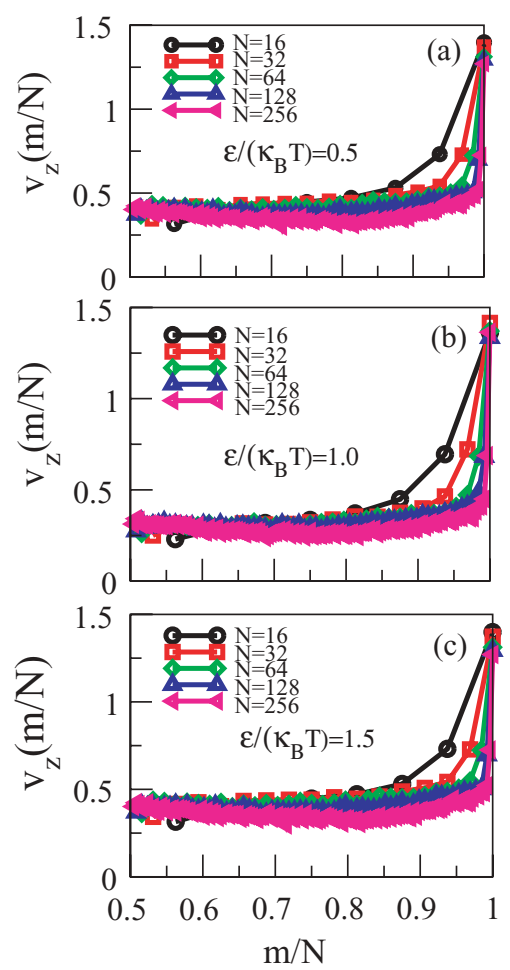

FIG. 8. (Color online) Average velocity on the monomer beads inside the pore as a function of the monomer index for chain lengths $N=16,32,64,128$, and 256, respectively, for (a) $\varepsilon / k_{B} T=0.5$, (b) $\varepsilon / k_{B} T=1.0$, and (c) $\varepsilon / k_{B} T=1.5$. The symbols have the same meaning as in Fig. 5. We note that in the large $N$ limit, the velocity of the monomers becomes almost constant except for the last few monomers. 


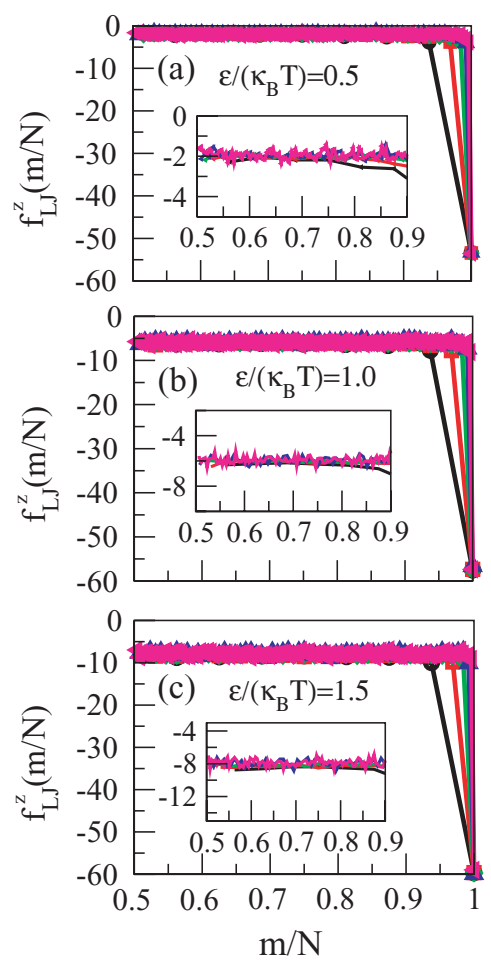

FIG. 9. (Color online) Average force on the monomer beads inside the pore as a function of the monomer index for chain lengths $N=16,32,64,128$, and 256 , respectively, for (a) $\varepsilon / k_{B} T=0.5$, (b) $\varepsilon / k_{B} T=1.0$, and (c) $\varepsilon / k_{B} T=1.5$. The symbols have the same meaning as in Fig. 4.

average value of $W(m)$ for $N / 2<m<N-4$ for each chain length. It is not hard to imagine from the data presented for chain lengths $N=16-256$ that the variation of $W(m)$ will become very small for long chains. The location of this peak $m_{\max }$ is an increasing function of chain length $N$ for a given interaction strength and vice versa. The insets of Fig. 7 show these aspects. The qualitative feature of this nonmonotonic dependence of the residence time on the monomer index is similar to the case of forced translocation [22] as well as to the case of translocation by a pulling force through a nanopore [26]. In both the cases the waiting time exhibits a maximum. In the case of forced translocation this is due to high density (jamming) of the segments just translocated but yet to equilibrate in the vicinity of the pore. In the case of pulling, the maximum occurs when the entropy of the pulled segment is equal to the entropy of the chain on the cis side. Our case is closer in analogy to the pulling case, which also has a wider flat region that is a characteristic of a single file process. For the case studied here the maximum occurs when the the globule is big enough so that it just balances the entropy of the self-avoiding chain on the $c i$ side. However, the maximum is weak for longer chains as there is no jamming on the trans side as the translocated segment immediately becomes part of the globule. We will notice in Sec. III C 4 that this results in a faster translocation than the corresponding driven case. Once a globule is formed on the trans side, the pulling force exerted on the monomer inside the pore by the globule is roughly balanced by the entropic force until the last few beads are left on the $c i s$ side. One other aspect in Fig. 7 worth mentioning is the sharp

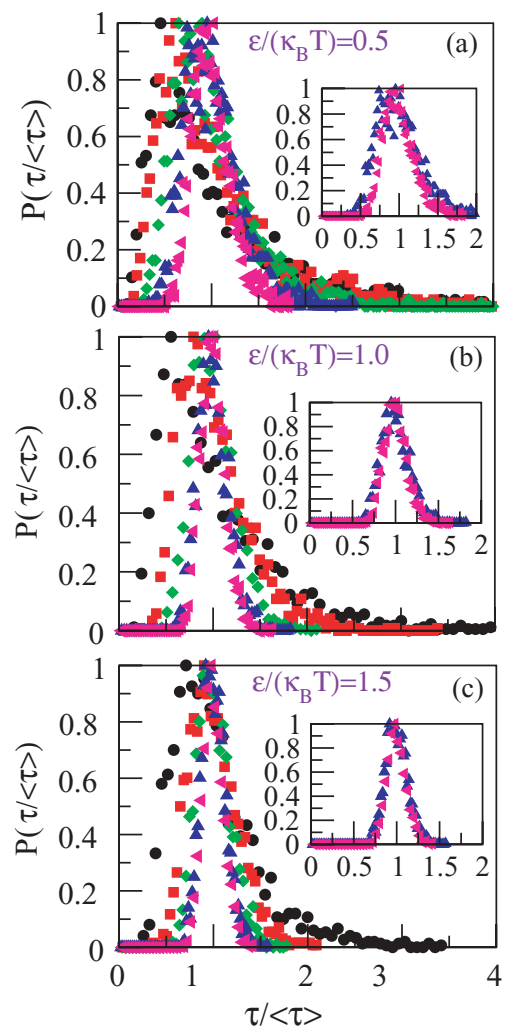

FIG. 10. (Color online) Scaled histograms for the MFPT for different chain lengths $N=16,32,64,128$, and 256 for (a) $\varepsilon / k_{B} T=$ 0.5 , (b) $\varepsilon / k_{B} T=1.0$, and (c) $\varepsilon / k_{B} T=1.5$ for $N_{t r}(t=0) / N=0.5$. The symbols have the same meaning as in Fig. 4. In general, for these values of $\varepsilon / k_{B} T$ we do not notice universal scaling for all chain length $N$. However, for longer chains and larger attraction strengths [insets in (a), (b), and (c)], we begin to see scaling emerges quite clearly.

fall of the residence time for $m / N<x_{\text {trans }}(t=0)$. These are the monomers originally placed at the trans side (please note that, with respect to Fig. 7, translocation occurs from right to the left) that enter the cis side due to back and forth motion of the chain. The probability of such events drops sharply as $m / N \ll x_{\text {trans }}(t=0)$, simply because beads originally at $t=0$ placed far away from the pore at the trans side do not reenter the pore. This explains also the sudden drop of the residence time for $m=N_{\text {trans }}(t=0)$. With increasing strength $\varepsilon / k_{B} T$ we find that this probability decreases (not shown here) even more sharply.

\section{Monomer velocity $v_{z}(m)$ and force $f_{\mathrm{LJ}}^{z}(m)$}

Since the residence time $W(m)$ exhibits a weak maximum one can guess from Fig. 7 that the velocity of the monomers while inside the bead will exhibit a weak minimum. This is shown in Figs. 8(a)-8(c). To a first approximation, we observe that a major fraction of the the monomers cross the pore with a near constant velocity except for the last few beads when they are pulled to the golube formed at the trans side. We have also looked at the longitudinal component of the corresponding force $f_{\mathrm{LJ}}^{z}(m)$ on the monomer $m$ inside the pore. But since the attractive part comes from the LJ part of the bad solvent, we have taken out the FENE part as it produces high frequency 


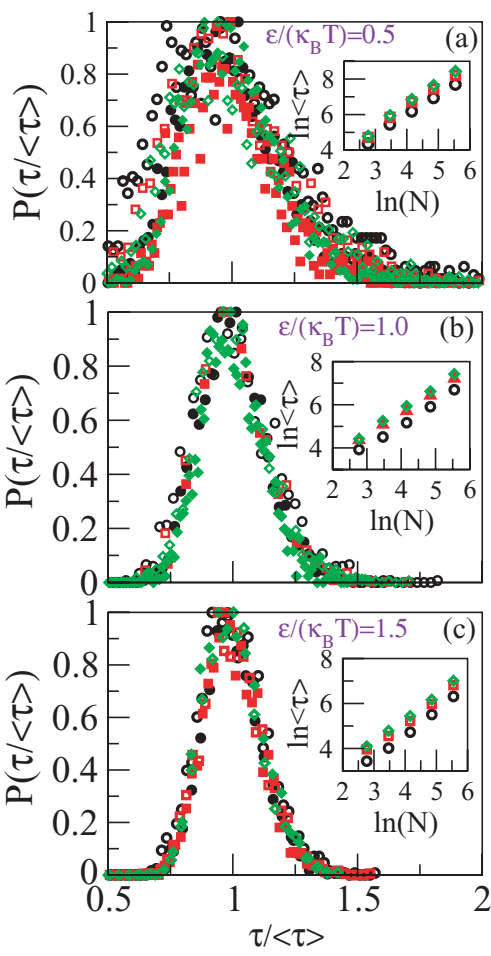

FIG. 11. (Color online) Scaled histograms for the MFPT for different chains $N=128$ (open symbols), and 256 (closed symbols) for three different values of the initial conditions for $x_{\text {trans }}(t=0)$ for (a) $\varepsilon / k_{B} T=0.5$, (b) $\varepsilon / k_{B} T=1.0$, and (c) $\varepsilon / k_{B} T=$ 1.5. The open(closed) circles, squares, and diamonds correspond to $x_{\text {trans }}(t=0)=0.5, x_{\text {trans }}(t=0)=0.25$, and $x_{\text {trans }}(t=0)=0.125$, respectively, for chain length 128(256). In each case the inset shows $\langle\tau\rangle \sim N$ for $x_{\text {trans }}(t=0)=0.5,0.25$, and 0.125 , respectively.

oscillations which are not important for this discussion. This force as shown in Figs. 9(a) through (c) is very flat except for the last few beads consistent with the behavior of the velocity of the monomers. The force on the last few monomers is large, but negative due to the deceleration when they become part of the globule at the trans side. We have also checked that the longitudinal velocity $v_{z}(m)$ and the force $f_{L J}^{z}(m)$ are proportional to each other.

\section{Distribution of translocation time \& translocation exponent}

Previous studies of driven translocation through a nanopore have revealed that histograms of the MFPT collapse on the same master curve. In this study we monitor translocation events having a fraction of the chain at the trans side at $t=$ 0 . Therefore, even if we assume that a power law relation holds for the mean translocation time on chain length $(\langle\tau\rangle \sim$ $N^{\alpha}$ ), due to the chosen initial condition (Fig. 1) we do not expect to see data collapse on a single master curve. However, by monitoring the trend of such a collapse as a function of decreasing fraction of monomers $x_{\text {trans }}(t=0)$ on the trans side (Fig. 1) we have been able to predict the trend. Figure 10 shows the histograms of the MFPT normalized by the $\langle\tau\rangle$ for three different values of the interaction strength for chain length $N=$ 16-256. We begin to see the trend for the data to collapse on the same master curve for larger values of $\frac{\epsilon}{k_{B} T}$ and also for longer chains $(N=128$ and 256$)$ only. To understand the limiting
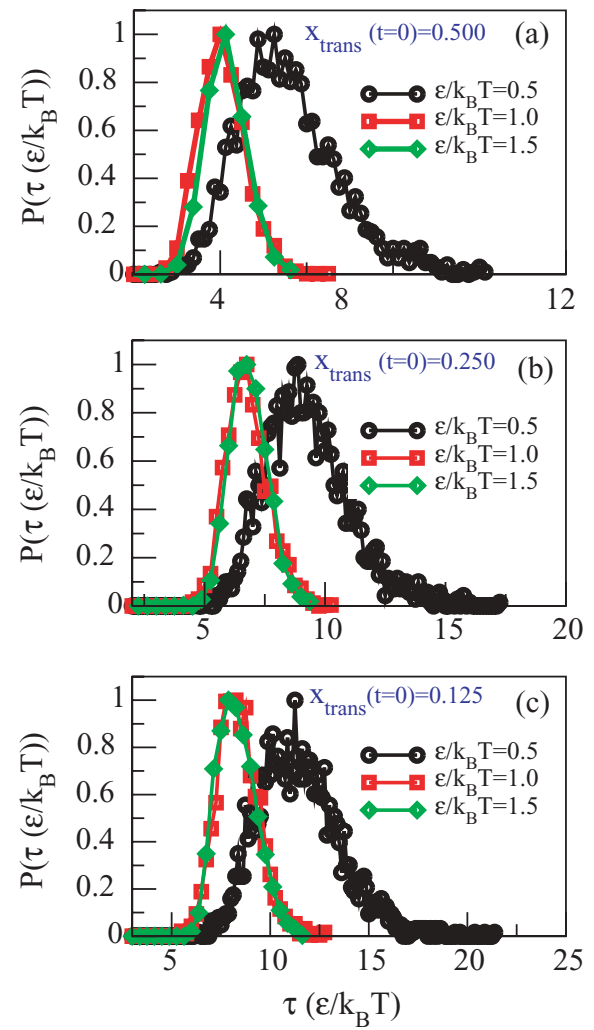

FIG. 12. (Color online) Scaled histograms for the MFPT for a $N=256$ chain. (a) $x_{\text {trans }}(t=0)=0.5$, (b) $x_{\text {trans }}(t=0)=0.25$, and (c) $x_{\text {trans }}(t=0)=0.125$ to check the dependence of $\langle\tau\rangle$ on $\varepsilon / k_{B} T$. In each figure we show the histogram for $\varepsilon / k_{B} T=0.5$ (circles), $\varepsilon / k_{B} T=1.0$ (squares), and $\varepsilon / k_{B} T=1.5$ (diamonds).

case $x_{\text {trans }}(t=0) \rightarrow 0$, in Fig. 11 we show the histograms as a function for $x_{\text {trans }}=0.5,0.25$, and 0.125 in for the two longest chains $N=128$ and 256 . This shows that the scaling function is independent of $x_{\text {trans }}(t=0)$. Therefore, from Figs. 10 and 11 we conclude that the translocation exponent extracted from these data will be the same as one would have obtained in the limit $x_{\text {trans }} \rightarrow 0$. We further notice that the data for $\frac{\epsilon}{k_{B} T}=1.0$ and 1.5 are very similar. The insets of Fig. 11 show $\langle\tau\rangle \sim N$ on a $\log$ scale. If we use the last three data points (for the reason that they collapse on the same master plot) we get $\alpha=$ $1.1 \pm 0.05$. It is worthwhile to note that the value $\alpha=1.1$ is lower than the numerical exponent for forced translocation [36] obtained for the same model. It signifies a faster translocation process.

We further extend our scaling analysis drawing analogy with the biased translocation. Previous studies of forced translocation have shown that [36] when the external bias $f_{\text {ext }}$ is small $\langle\tau\rangle \propto 1 / f_{\text {ext }}$, so that histograms for different bias when scaled by $\langle\tau\rangle f_{\text {ext }}$ collapse on the same master curve. In our studies the globule at the trans side pulls the rest of the chain at the cis side and therefore exerts a force on the monomer inside the pore. For clarity, in Fig. 12 we show these scaled histograms for $N=256$ only where we notice excellent data collapse for $\frac{\varepsilon}{k_{B} T}=1.0$ and 1.5. This implies that when we plot the scaled average translocation time $\langle\tau\rangle \cdot \frac{\epsilon}{k_{B} T}$ as a function of the chain length $N$ the data points will be the same within statistical error bars. Figure 13 confirms 

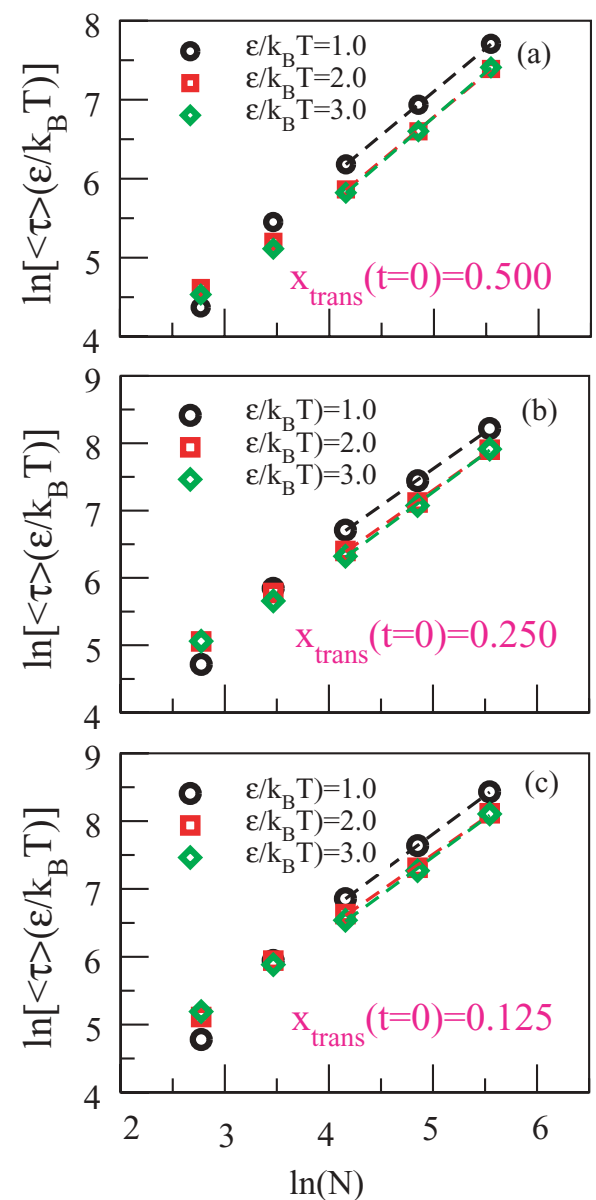

FIG. 13. (Color online) Variation of $\langle\tau\rangle \frac{\varepsilon}{k_{B} T}$ as a function of $N$ (log-log plot) for (a) $x_{\text {trans }}(t=0)=0.5$, (b) $x_{\text {trans }}(t=0)=0.25$, and (c) $x_{\text {trans }}(t=0)=0.125$. The symbols have the same meaning as in Fig. 11 .

that this is indeed the case for different initial conditions $x_{\text {trans }}(t=0)=0.5,0.25$, and 0.125 , respectively.

\section{Free energy analysis}

Let us now analyze the results in terms of an analytical expression for the free energy following Muthukumar [8]. For a translocating chain of length $N$ having $m$ translocated monomers at the trans side the free energy is given by [8]

$$
\frac{F(m)}{k_{B} T}=\left(1-\gamma_{\text {trans }}\right) \ln (m)+\left(1-\gamma_{\text {cis }}\right) \ln (N-m)+m \frac{\Delta \mu}{k_{B} T} \text {. }
$$

Here $\gamma_{\text {cis }}$ and $\gamma_{\text {trans }}=0.5, \simeq 0.69$, and 1 for Gaussian, selfavoiding, and rod-like chains, respectively [8,43]. For the case that we consider here, $\gamma_{c i s}=0.69$ and the first term is not present as the chain conformation at the trans side is not a random walk, rather it is a globule. The difference in the solvent conditions is put in through the $m \frac{\Delta \mu}{k_{B} T}$ term. In Fig. 14 we show two cases where (a) $N \Delta \mu=$ $0.1\left(1-\gamma_{\text {cis }}\right) \ln (N)$ and (b) where $N \Delta \mu=\left(1-\gamma_{\text {cis }}\right) \ln (N)$. In other words, we look at the cases where the net change in chemical potential after the translocation is either a small fraction of the total change in the entropy [Fig. 14(a)], or
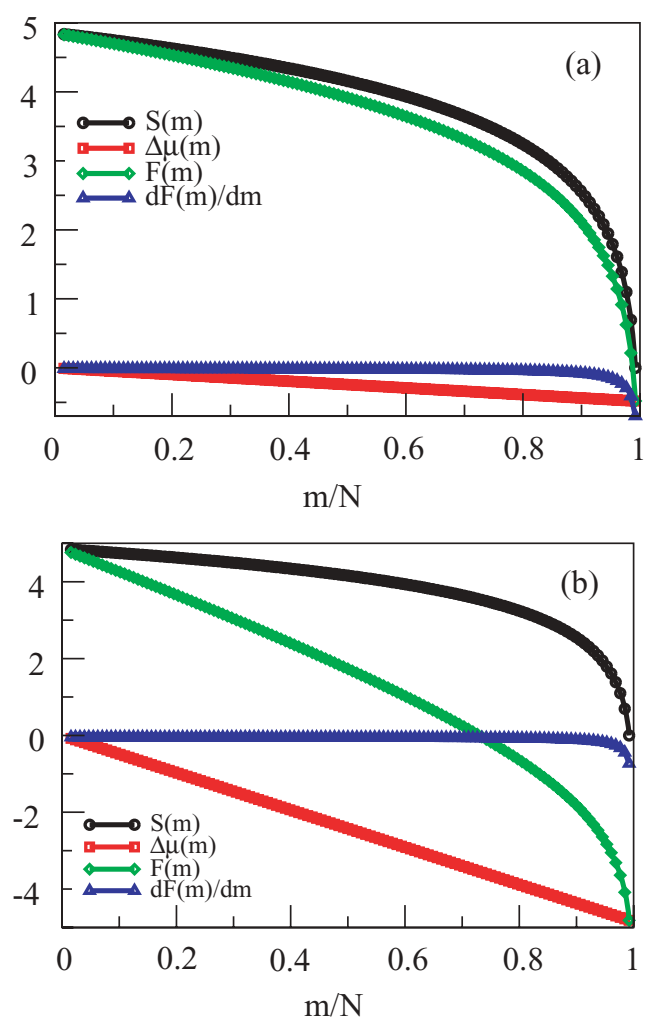

FIG. 14. (Color online) Free energy landscape using Eq. (8) for $\gamma_{c i s}=0.69$, and neglecting the first term as a function of the monomer fraction $m / N$; (a) for $N \Delta \mu=0.1\left(1-\gamma_{c i s}\right) \ln (N)$ and (b) for $N \Delta \mu=\left(1-\gamma_{\text {cis }}\right) \ln (N)$ in units of $k_{B} T$. The circles, squares, and diamonds represent the entropic part of the free energy, the chemical potential difference, and the total free energy $F(m)$; the blue triangles represent $d F(m) / d m$.

comparable [Fig. 14(b)]. In the same figure we also plot the derivative of the total free energy $\partial F(m) / \partial m$. Notice that in either case the drop in $\Delta \mu$ is always linear. Therefore, the rapid change in the free energy near the end of the translocation process is predominantly due to the entropic term. We also note that the derivative of the free energy, which is proportional to the velocity or the force, is relatively flat for a large fraction of the translocation process and largely insensitive to this ratio of entropy and chemical potential difference. This free energy landscape, which produces a relatively flat velocity profile, essentially explains our simulation results.

\section{SUMMARY}

We studied polymer translocation through a narrow pore induced by different conformation statistics at the cis and trans side of the pore. Specifically, we studied translocation induced by a bad solvent at the trans side. The trans segments of the polymer form a globule which acts as a ratchet eventually forcing the chain to translocate from the cis to the trans side. We check the similarities and differences of this conformation-induced translocation with that of a driven translocation where there is a bias inside the pore, and with the case where the polymer chain is pulled at one end. We analyze the results using the free energy proposed by Muthukumar. We find the free energy eventually goes downhill, but the 
derivative of the free energy with respect to the monomer index is relatively flat except for the last few monomers, where the free energy is dominated by entropy. This flatness of the free energy derivative qualitatively explains the behavior of the longitudinal components of the velocity and force, respectively, for the monomer inside the pore.

We find that $\langle\tau\rangle \sim N^{1.1 \pm 0.05}$ and $\langle\tau\rangle \propto\left(\varepsilon / k_{B} T\right)^{-1}$ for $\varepsilon / k_{B} T \geqslant 1.0$, so that the histograms $P\left(\tau \cdot \frac{\varepsilon}{k_{B} T}\right)$ as a function of $\tau \cdot \frac{\varepsilon}{k_{B} T}$ collapse on the same master curve (Fig. 12). We find the translocation exponent $\alpha \simeq 1.1 \pm 0.05$ is lower than that of the case of forced translocation [44]. This we attribute due to the absence of jamming in the trans side where the bad solvent favors the incoming monomer to be readily adsorbed to the preexisting globule in the vicinity of the pore (Figs. 3 and 4). Since our results are independent of different initial conditions $x_{\text {trans }}(t=0)=0.25$ and $x_{\text {trans }}(t=0)=0.125$ for longer chain lengths, we believe that in the limit $x_{\text {trans }}(t=0) \rightarrow 0$ these results will remain valid. The physical picture that emerges is that the chain makes a single file translocation where the monomers inside the pore move with constant velocity proportional to the pulling force produced by the globule. In that sense, this case bears more similarity to that case where the polymer is pulled at one end. Various aspects of translocation of biomolecules through a narrow pore across a membrane continue to offer interesting and important problems. Currently we are looking into translocation of a random heteropolymer driven by asymmetric solvent conditions, which we will report in a separate publication.

\section{ACKNOWLEDGMENTS}

C.L. at the University of Central Florida has been supported by the Florida Education Fund (McKnight Doctoral Fellowship). A.B. acknowledges partial support through NSFCHE Grant No. 0809821 and funds from the Department of Applied Physics, Aalto University School of Science for a visiting Professorship. T.A.N. acknowledges a travel fund from the University of Central Florida and funds by the Academy of Finland through the COMP CoE and TransPoly consortium grants. A.B. acknowledges fruitful discussion with Kurt Binder. We thank the anonymous referees for their constructive comments on the manuscript.
[1] B. Alberts et al., Molecular Biology of the Cell (Garland Publishing, New York, 1994).

[2] J. J. Kasianowitch, E. Brandin, D. Branton, and D. Deamer, Proc. Natl. Acad. Sci. USA 93, 13770 (1996); A. Meller, L. Nivon, E. Brandin, J. Golovchenko, and D. Branton, ibid. 97, 1097 (2000).

[3] A. Meller, J. Phys. Condens. Matter 15, R581 (2003).

[4] M. Muthukumar, Annu. Rev. Biophys. Biomol. Struct. 36, 435 (2007).

[5] J. L. Li, M. Gershow, D. Stein, E. Brandin, and J. A. Golovchenko, Nat. Mater. 2, 611 (2003); A. J. Storm, J. H. Chen, X. S. Ling, H. W. Zandbergen, and C. Dekker, ibid. 2, 537 (2003).

[6] W. Sung and P. J. Park, Phys. Rev. Lett. 77, 783 (1996).

[7] P. J. Park and W. Sung, J. Chem. Phys. 108, 3013 (1998).

[8] M. Muthukumar, J. Chem. Phys. 111, 10371 (1999).

[9] M. Muthukumar, Phys. Rev. Lett. 86, 3188 (2001).

[10] J. Chuang, Y. Kantor, and M. Kardar, Phys. Rev. E 65, 011802 (2001).

[11] Y. Kantor and M. Kardar, Phys. Rev. E 69, 021806 (2004).

[12] J. L. A. Dubbeldam, A. Milchev, V. G. Rostiashvili, and T. A. Vilgis, Phys. Rev. E 76, 010801(R) (2007).

[13] J. L. A. Dubbeldam, A. Milchev, V. G. Rostiashvili, and T. A. Vilgis, Europhys. Lett. 79, 18002 (2007).

[14] J. L. A. Dubbeldam, A. Milchev, V. G. Rostiashvili, and T. A. Vilgis, J. Phys. Condens. Matter 21, 098001 (2009).

[15] J. K. Wolterink, G. T. Barkema, and D. Panja, Phys. Rev. Lett. 96, 208301 (2006).

[16] D. Panja, G. T. Barkema, and R. C. Ball, J. Phys. Condens. Matter 19, 432202 (2007); 20, 075101 (2008).

[17] H. Vocks, D. Panja, G. T. Barkema, and R. C. Ball, J. Phys. Condens. Matter 20, 095224 (2008).

[18] T. Sakaue, Phys. Rev. E 76, 021803 (2007); 81, 041808 (2010).
[19] M. G. Gauthier and G. W. Slater, J. Chem. Phys. 128, 065103 (2008); 128, 205103 (2008).

[20] A. Milchev, K. Binder, and A. Bhattacharya, J. Chem. Phys. 121, 6042 (2004).

[21] K. Luo, T. Ala-Nissila, and S.-C. Ying, J. Chem. Phys. 124, 034714 (2006).

[22] I. Huopaniemi, K. Luo, T. Ala-Nissila, and S.-C. Ying, J. Chem. Phys. 125, 124901 (2006).

[23] K. Luo, I. Huopaniemi, T. Ala-Nissila, and S.-C. Ying, J. Chem. Phys. 124, 114704 (2006).

[24] K. Luo, T. Ala-Nissila, S.-C. Ying, and A. Bhattacharya, J. Chem. Phys. 126, 145101 (2007).

[25] K. F. Luo, T. Ala-Nissila, S.-C. Ying, and A. Bhattacharya, Phys. Rev. Lett. 99, 148102 (2007).

[26] I. Huopaniemi, K. F. Luo, T. Ala-Nissila, and S.-C. Ying, Phys. Rev. E 75, 061912 (2007).

[27] K. F. Luo, T. Ala-Nissila, S.-C. Ying, and A. Bhattacharya, Phys. Rev. Lett. 100, 058101 (2008).

[28] K. Luo Santtu, T. T. Ollila, I. Huopaniemi, T. Ala-Nissila, P. Pomorski, M. Karttunen, S.-C. Ying, and A. Bhattacharya, Phys. Rev. E 78, 050901(R) (2008).

[29] K. Luo, T. Ala-Nissila, S.-C. Ying, and R. Metzler, Europhys. Lett. 88, 68006 (2009).

[30] D. Wei, W. Yang, X. Jin, and Q. Liao, J. Chem. Phys. 126, 204901 (2007).

[31] S. Matysiak, A. Montesi, M. Pasquali, A. B. Kolomeisky, and C. Clementi, Phys. Rev. Lett. 96, 118103 (2006).

[32] S. Guillouzic and G. W. Slater, Phys. Lett. A 359, 261 (2006).

[33] M. G. Gauthier and G. W. Slater, Eur. Phys. J. E 25, 17 (2008).

[34] M. G. Gauthier and G. W. Slater, Phys. Rev. E 79, 021802 (2009).

[35] V. V. Lehtola, R. P. Linna, and K. Kaski, Europhys. Lett. 85, 58006 (2009); Phys. Rev. E 78, 061803 (2008).

[36] A. Bhattacharya, W. Morrison, K. Luo, T. Ala-Nissila, S.-C. Ying, A. Milchev, and K. Binder, Eur. Phys. J. E 29, 423 (2009). 
[37] A. Bhattacharya and K. Binder, Phys. Rev. E 81, 041804 (2010).

[38] P. G. de Gennes, Scaling Concepts in Polymer Physics (Cornell University Press, Ithaca, NY, 1979); M. Doi and S. F. Edwards, The Theory of Polymer Dynamics (Oxford University Press, Oxford, 1986).

[39] S. Simon, C. Peskin, and G. Oster, Proc. Natl. Acad. Sci. USA 89, 3770 (1992).

[40] R. Zandi, D. Reguera D, J. Rudnick, and G. M. Gelbart, Proc. Natl. Acad. Sci. USA 100, 8649 (2003).

[41] G. S. Grest and K. Kremer, Phys. Rev. A 33, 3628 (1986).
[42] The $\Theta$ temperature for the parameters used in this work has already been determined. For example, please see H. Liu, A. Bhattacharya, and A. Chakrabarti, J. Chem. Phys. 111, 11183 (1999).

[43] E. Eisenriegler, K. Kremer, and K. Binder, J. Chem. Phys. 77, 6296 (1982).

[44] For the same model for the case of forced translocation the exponent $\alpha=1.34$ [A. Bhattacharya, W. Morrison, K. Luo, T. Ala-Nissila, S.-C. Ying, A. Milchev, and K. Binder, Eur. Phys. J. E 29, 423 (2009)]. 